\title{
Optimal Control of a Delayed HIV Infection Model via Fourier Series
}

\author{
Gh. Ghanbari ${ }^{1}$ and M. H. Farahi ${ }^{1,2}$ \\ ${ }^{1}$ Department of Applied Mathematics, Ferdowsi University of Mashhad, Mashhad 9177948953, Iran \\ ${ }^{2}$ The Center of Excellence on Modelling and Control Systems (CEMCS), Mashhad 9177948953, Iran \\ Correspondence should be addressed to Gh. Ghanbari; ghodsiye.ghanbari@gmail.com
}

Received 27 May 2014; Revised 13 August 2014; Accepted 14 August 2014; Published 26 August 2014

Academic Editor: Giovanni P. Galdi

Copyright (C) 2014 Gh. Ghanbari and M. H. Farahi. This is an open access article distributed under the Creative Commons Attribution License, which permits unrestricted use, distribution, and reproduction in any medium, provided the original work is properly cited.

\begin{abstract}
We present a delayed optimal control which describes the interaction of the immune system with the human immunodeficiency virus $(\mathrm{HIV})$ and $\mathrm{CD} 4^{+} \mathrm{T}$-cells. In order to improve the therapies, treatment and the intracellular delays are incorporated into the model. The optimal control in this model represents the efficiency of drug treatment in preventing viral production and new infections. The optimal pair of control and trajectories of this nonlinear delay system with quadratic cost functional is obtained by Fourier series approximation. The method is based on expanding time varying functions in the nonlinear delay system into their Fourier series with unknown coefficients. Using operational matrices for integration, product, and delay, the problem is reduced to a set of nonlinear algebraic equations.
\end{abstract}

\section{Introduction}

Delays occur frequently in biological, chemical, electronic, and transportation systems [1]. Many mathematical models have been developed in order to understand the dynamics of HIV infection [2-7]. Moreover, optimal control methods have been applied to the derivation of optimal therapies for this HIV infection [8-14]. All these methods are based on HIV models which ignore the intracellular delay by assuming that the infectious process is instantaneous; that is, as soon as the virus enters an uninfected cell, it starts to produce virus particles; however, this is not reasonable biologically. In this paper, we consider the mathematical model of HIV infection with intracellular delay presented in [15] in order to make the model more tangible and closer to what happens in reality.

Orthogonal functions $(\mathrm{OFs})$ have received considerable attention in dealing with various problems of dynamic systems. Using operational matrices, the technique is based on reduction of these problems to systems of algebraic equations. Special attention has been given to applications of Walsh functions [16], block-pulse functions [17], Laguerre polynomials [18], Legendre polynomials [19], Chebyshev polynomials [20], and Fourier series [21].
In this paper, we apply Fourier series approximation to find the optimal pair of control and trajectories of the nonlinear delayed optimal control system governed by ordinary delay differential equations which describe the interaction of the human immunodeficiency virus (HIV). Operational matrices of integration, product, and delay have the most important role in our method. The paper is organized as follows. Section 2 consists of an introduction to Fourier series approximation and operational and other matrices, being used in Section 4. Section 3 proposes the delayed optimal control model of HIV infection. In Section 4, we utilize the Fourier series approximation to solve our model and results are demonstrated by some figures. Finally, the conclusions are summarized in Section 5.

\section{Properties of Fourier Series}

A measurable function defined over the interval 0 to $L$ may be expanded into a Fourier series as follows:

$$
f(t)=a_{0}+\sum_{n=1}^{\infty}\left\{a_{n} \cos \left(\frac{2 n \pi t}{L}\right)+a_{n}^{*} \sin \left(\frac{2 n \pi t}{L}\right)\right\}
$$


where the Fourier coefficients $a_{n}$ and $a_{n}^{*}$ are given by

$$
\begin{gathered}
a_{0}=\frac{1}{L} \int_{0}^{L} f(t) \mathrm{d} t, \\
a_{n}=\frac{2}{L} \int_{0}^{L} f(t) \cos \left(\frac{2 n \pi t}{L}\right) \mathrm{d} t, \quad n=1,2,3, \ldots, \\
a_{n}^{*}=\frac{2}{L} \int_{0}^{L} f(t) \sin \left(\frac{2 n \pi t}{L}\right) \mathrm{d} t, \quad n=1,2,3, \ldots
\end{gathered}
$$

The series in (1) has an infinite number of terms. To obtain an approximate expression for $f(t)$, one can truncate the series up to the $(2 r+1)$ th term as follows:

$$
\begin{aligned}
f(t) & =a_{0}+\sum_{n=1}^{r}\left\{a_{n} \cos \left(\frac{2 n \pi t}{L}\right)+a_{n}^{*} \sin \left(\frac{2 n \pi t}{L}\right)\right\} \\
& =A^{T} \phi(t),
\end{aligned}
$$

where the Fourier series coefficient vector $A$ and the Fourier series vector $\phi(t)$ are defined as

$$
\begin{gathered}
A=\left[\begin{array}{lllllllll}
a_{0} & a_{1} & a_{2} & \ldots & a_{r} & a_{1}^{*} & a_{2}^{*} & \ldots & a_{r}^{*}
\end{array}\right]^{T}, \\
\Phi=\left[\begin{array}{lllllll}
\phi_{0}(t) & \phi_{1}(t) & \ldots & \phi_{r}(t) & \phi_{1}^{*}(t) & \ldots & \phi_{r}^{*}(t)
\end{array}\right]^{T},
\end{gathered}
$$

where

$$
\begin{gathered}
\phi_{n}(t)=\cos \left(\frac{2 n \pi t}{L}\right), \quad n=0,1,2,3, \ldots, r, \\
\phi_{n}^{*}(t)=\sin \left(\frac{2 n \pi t}{L}\right), \quad n=1,2,3, \ldots, r .
\end{gathered}
$$

The elements of $\Phi(t)$ are orthogonal in the interval $[0, L]$. By integrating both sides of (6a) and (6b) with respect to $\theta$, we obtain

$$
\begin{gathered}
\int_{0}^{t} \phi_{0}(\theta) \mathrm{d} \theta=t, \\
\int_{0}^{t} \phi_{n}(\theta) \mathrm{d} \theta=\frac{L}{2 n \pi}\left[\phi_{n}^{*}(t)-\phi_{n}^{*}(0)\right], \quad n \geq 1, \\
\int_{0}^{t} \phi_{n}^{*}(\theta) \mathrm{d} \theta=\frac{L}{2 n \pi}\left[\phi_{n}(0)-\phi_{n}(t)\right], \quad n \geq 1 .
\end{gathered}
$$

Now, we approximate the function $t$ in the (7) by a truncated Fourier series. Consequently, the forward integral of the Fourier series vector $\Phi(t)$ can be represented by

$$
\int_{0}^{t} \Phi(\theta) \mathrm{d} \theta \cong P \Phi(t)
$$

where $P_{(2 r+1) \times(2 r+1)}$ is the Fourier series operational matrix of forward integration and is given as

$$
P=L\left[\begin{array}{ccccccccccc}
\frac{1}{2} & 0 & 0 & \cdots & 0 & 0 & \frac{-1}{\pi} & \frac{-1}{2 \pi} & \cdots & \frac{-1}{(r-1) \pi} & \frac{-1}{r \pi} \\
0 & 0 & 0 & \cdots & 0 & 0 & \frac{1}{2 \pi} & 0 & \cdots & 0 & 0 \\
\vdots & \vdots & \vdots & & \vdots & \vdots & \vdots & \vdots & & \vdots & \vdots \\
0 & 0 & 0 & \cdots & 0 & 0 & 0 & 0 & \cdots & \frac{1}{2(r-1) \pi} & 0 \\
0 & 0 & 0 & \cdots & 0 & 0 & 0 & 0 & \cdots & 0 & \frac{1}{2 r \pi} \\
\frac{1}{2 \pi} & \frac{-1}{2 \pi} & 0 & \cdots & 0 & 0 & 0 & 0 & \cdots & 0 & 0 \\
\frac{1}{4 \pi} & 0 & \frac{-1}{4 \pi} & \cdots & 0 & 0 & 0 & 0 & \cdots & 0 & 0 \\
\vdots & \vdots & \vdots & & \vdots & \vdots & \vdots & \vdots & & \vdots & \vdots \\
\frac{1}{2 r \pi} & 0 & 0 & \cdots & 0 & \frac{-1}{2 r \pi} & 0 & 0 & \cdots & 0 & 0
\end{array}\right] .
$$


As a result, we obtain

$$
\int_{\tau}^{t} \Phi(\theta-\tau) \mathrm{d} \theta=P \Phi(t-\tau)
$$

Moreover,

$$
\int_{0}^{\tau} \Phi(t) \mathrm{d} t=\Phi(\tau)-\Phi(0)=Z \Phi(t),
$$

where

$$
Z=\left[\begin{array}{ccccccc}
\tau & 0 & \cdots & 0 & 0 & \cdots & 0 \\
\frac{L}{2 \pi} \sin \frac{2 \pi \tau}{L} & 0 & \cdots & 0 & 0 & \cdots & 0 \\
\vdots & \vdots & & \vdots & \vdots & & \vdots \\
\frac{L}{2 r \pi} \sin \frac{2 r \pi \tau}{L} & 0 & \cdots & 0 & 0 & \cdots & 0 \\
\frac{L}{2 \pi}\left(1-\cos \frac{2 \pi \tau}{L}\right) & 0 & \cdots & 0 & 0 & \cdots & 0 \\
\vdots & \vdots & & \vdots & \vdots & & \vdots \\
\frac{L}{2 r \pi}\left(1-\cos \frac{2 r \pi \tau}{L}\right) & 0 & \cdots & 0 & 0 & \cdots & 0
\end{array}\right] .
$$

The delay function $\Phi(t-\tau)$ is the shift of the function $\Phi(t)$ defined in (5) along the time axis by $\tau$. The general expression is given by

$$
\Phi(t-\tau)=S_{\tau} \Phi(t)
$$

where $S_{\tau}$ is the delay operational matrix of Fourier series, which is as follows:

$$
S_{\tau}=\left[\begin{array}{ccccccccc}
1 & 0 & 0 & \cdots & 0 & 0 & 0 & \cdots & 0 \\
0 & \cos \frac{2 \pi}{L} & 0 & \cdots & 0 & \sin \frac{2 \pi}{L} & 0 & \cdots & 0 \\
0 & 0 & \cos \frac{4 \pi}{L} & \cdots & 0 & 0 & \sin \frac{4 \pi}{L} & \cdots & 0 \\
\vdots & \vdots & \vdots & \ddots & \vdots & \vdots & \vdots & \ddots & \vdots \\
0 & -\sin \frac{2 \pi}{L} & 0 & \cdots & 0 & \cos \frac{2 \pi}{L} & 0 & \cdots & 0 \\
0 & 0 & -\sin \frac{4 \pi}{L} & \cdots & 0 & 0 & \cos \frac{4 \pi}{L} & \cdots & 0 \\
\vdots & \vdots & \vdots & \ddots & \vdots & \vdots & \vdots & \ddots & \vdots \\
0 & 0 & 0 & \cdots & -\sin \frac{2 r \pi}{L} & 0 & 0 & \cdots & \cos \frac{2 r \pi}{L}
\end{array}\right] .
$$

Also, we have (see [21])

$$
\begin{aligned}
& D=\int_{0}^{L} \Phi(t) \Phi^{T}(t) \mathrm{d} t=L\left[\begin{array}{llllll}
1 & & & & & \\
& \frac{1}{2} & & & & \\
& & \frac{1}{2} & & & \\
& & \frac{1}{2} & & \\
& & \frac{1}{2} & & \\
& 0 & & & \ddots & \\
& & & & \frac{1}{2}
\end{array}\right], \\
& W=\int_{0}^{L} \Phi(t) \mathrm{d} t=L\left[\begin{array}{c}
1 \\
0 \\
\vdots \\
0
\end{array}\right] \text {. }
\end{aligned}
$$

Using (3) and (10) leads to

$$
\int_{0}^{t} f(\theta) \mathrm{d} \theta \cong \int_{0}^{t} A^{T} \Phi(\theta) \mathrm{d} \theta \cong A^{T} P \Phi(t)
$$

We can get

$$
\Phi(t) \Phi^{T}(t) A=\widetilde{A} \Phi(t),
$$

where $\widetilde{A}$ is the product operational matrix for the vector $A$ (see [22]).

As a result,

$$
\Phi(t-\tau) \Phi^{T}(t-\tau) A=\widetilde{A} \Phi(t-\tau)
$$




\section{HIV Delayed Optimal Control Problem}

Using time delay dynamic systems, many physical systems are best modeled as follows:

$$
\begin{gathered}
\frac{d x(t)}{d t}=F\left(t, x(t), u(t), x_{1}\left(t-\tau_{1}\right), \ldots, x_{p}\left(t-\tau_{p}\right)\right. \\
\left.u_{1}\left(t-\eta_{1}\right), \ldots, u_{m}\left(t-\eta_{m}\right)\right), \quad t \in\left[t_{0}, t_{f}\right] \\
x(t)=\Phi(t), \quad t \leq t_{0}, \\
u(t)=\Psi(t), \quad t \leq t_{0}
\end{gathered}
$$

where the state $x(t)$ is a $p$ vector function, $u(t)$ is an $m$ vector control function, $\tau_{i}$ and $\eta_{j}(i=1,2, \ldots, p, j=1,2, \ldots, m)$ are nonnegative constant time delays, and the vector functions $\Phi(t)$ and $\Psi(t)$ are defined appropriately and given (see [2326]).

In some systems of this type, it is desirable to select the optimal pair $\left(x^{*}(\cdot), u^{*}(t)\right)$ which satisfies $(22 \mathrm{a}),(22 \mathrm{~b})$, and (22c) and minimizes performance criterion modeled by a cost function of the form

$$
I=G\left(x\left(t_{f}\right), t_{f}\right)+\int_{t_{0}}^{t_{f}} g(t, x(t), u(t)) \mathrm{d} t .
$$

The following model is a delayed control system of HIV infection of $\mathrm{CD} 4^{+} \mathrm{T}$-cells (for more details, see [15]):

$$
\begin{gathered}
\dot{T}=s-\mu_{T} T(t)+r T(t)\left(1-\frac{T(t)+I(t)}{T_{\max }}\right) \\
-K_{1} V(t) T(t), \\
\dot{I}=K_{2} V(t-1) T(t-1)-\mu_{I} I(t), \\
\dot{V}=N_{1} \mu_{b} I(t) u(t)-K_{1} V(t) T(t)-\mu_{V} V(t), \\
T(t)=120, \quad t \in[-1,0], \\
I(t)=25, \quad t \in[-1,0], \\
V(t)=2000, \quad t \in[-1,0], \\
u(t)=0, \quad t \in[-1,0], \\
0 \leq u(t) \leq 1, \quad t \in\left[t_{0}, t_{f}\right],
\end{gathered}
$$

where control function $u(t)$ is showing the effect of drugs on HIV virus production. For the delayed control model (24a), (24b), (24c), (24d), (24e), (24f), (24g), and (24h), we consider the objective functional to be defined as

$$
J(T, u)=\int_{t_{0}}^{t_{f}}\left(T-\frac{1}{2} B(1-u(t))^{2}\right) \mathrm{d} t,
$$

where $B$, the weight of current costs of treatment, is assumed to be 100 . Our goal is to maximize the objective functional (25) subject to the delayed control system (24a), (24b), (24c), (24d), (24e), (24f), (24g), and (24h), that is, to maximize the total count of $\mathrm{CD} 4^{+}$T-cells and to minimize the costs of treatment. The treatment interval is assumed to be $I=$ $\left[t_{0}, t_{f}\right]=[0,300]$, which shows the duration on treatment in terms of days.

\section{Solving the Delayed Optimal Control Model of the HIV Infection Using Fourier Series}

According to Section 2, each function of $T(\cdot), I(\cdot), V(\cdot)$, and $U(\cdot)$ in the interval $I$ can be approximated by a Fourier series. So,

$$
\begin{gathered}
T(t)=\widehat{T}^{T} \Phi(t)=\Phi^{T}(t) \widehat{T}, \\
I(t)=\widehat{I}^{T} \Phi(t)=\Phi^{T}(t) \widehat{I}, \\
V(t)=\widehat{V}^{T} \Phi(t)=\Phi^{T}(t) \widehat{V}, \\
u(t)=\widehat{U}^{T} \Phi(t)=\Phi^{T}(t) \widehat{U},
\end{gathered}
$$

where $\widehat{T}, \widehat{I}, \widehat{V}$, and $\widehat{U}$ are, respectively, the Fourier series coefficient vectors (all are unknown) of $T(t), I(t), V(t)$, and $u(t)$. For time delay functions $T(t-1)$ and $V(t-1)$, we have

$$
\begin{gathered}
0 \leq t \leq 1 \Longrightarrow-1 \leq t-1 \leq 0, \\
T(t-1)=120, \quad 0 \leq t \leq 1, \\
V(t-1)=2000, \quad 0 \leq t \leq 1 .
\end{gathered}
$$

Therefore, for the interval $I$, we have

$$
\begin{gathered}
T(t-1)= \begin{cases}120, & 0 \leq t \leq 1, \\
\widehat{T}^{T} \Phi(t-1) & 1 \leq t \leq 300,\end{cases} \\
V(t-1)= \begin{cases}2000, & 0 \leq t \leq 1, \\
\widehat{V}^{T} \Phi(t-1), & 1 \leq t \leq 300 .\end{cases}
\end{gathered}
$$

By using (17), (18), and (26), one can rewrite (25) as follows:

$$
\begin{aligned}
& J\left(T, u_{2}\right) \\
& =\int_{0}^{300}\left[\widehat{T}^{T} \Phi(t)-50\right. \\
& \left.\quad \times\left(A^{T} \Phi(t)+\widehat{U}^{T} \Phi(t) \Phi^{T}(t) \widehat{U}-2 \widehat{U}^{T} \Phi(t)\right)\right] \mathrm{d} t \\
& =\widehat{T}^{T} W-50\left(A^{T} W+\widehat{U}^{T} D \widehat{U}-2 \widehat{U}^{T} W\right),
\end{aligned}
$$

where $A^{T} \Phi(t)$ is Fourier series of 1 , while $A=[1,0, \ldots, 0$, $0, \ldots, 0]^{T}$. 
By integrating both sides of (24a) from 0 to $t$ and using (26), (20), and (10), we obtain

$$
\begin{gathered}
\int_{0}^{t} \dot{T}(\theta) \mathrm{d} \theta=T(t)-T(0)=\widehat{T}^{T} \Phi(t)-T_{0}^{T} \Phi(t) \\
\int_{0}^{t}\left[s-\mu_{T} T+r T\left(1-\frac{T+I}{T_{\max }}\right)-K_{1} V T\right] \mathrm{d} \theta \\
=\int_{0}^{t}\left[S^{T} \Phi(\theta)-\mu_{T} \widehat{T}^{T} \Phi(\theta)+r \widehat{T}^{T} \Phi(\theta)-\frac{r}{T_{\max }}\right. \\
\quad \times\left(\widehat{T}^{T} \Phi(\theta) \Phi^{T}(\theta) \widehat{T}+\widehat{T}^{T} \Phi(\theta) \Phi^{T}(\theta) \widehat{I}\right) \\
\left.\quad-K_{1} \widehat{V}^{T} \Phi(\theta) \Phi^{T}(\theta) \widehat{T}\right] \mathrm{d} \theta \\
=S^{T} P \Phi(t)+\left(r-\mu_{T}\right) \widehat{T}^{T} P \Phi(t) \\
-\frac{r}{T_{\max }}\left(\widehat{T}^{T} \widetilde{T} P \Phi(t)+\widehat{T}^{T} \widetilde{I} P \Phi(t)\right) \\
-K_{1} \widehat{V}^{T} \widetilde{T} P \Phi(t)
\end{gathered}
$$

where $S$ and $T_{0}$ are the Fourier series coefficient vectors of $s$ and $T(0)$, respectively; that is, $S=[s, 0, \ldots, 0,0, \ldots, 0]^{T}, T_{0}=$ $[120,0, \ldots, 0,0, \ldots, 0]^{T}$.

So,

$$
\begin{aligned}
\widehat{T}^{T} \Phi(t)-T_{0}^{T} \Phi(t) \\
=S^{T} P \Phi(t)+\left(r-\mu_{T}\right) \widehat{T}^{T} P \Phi(t) \\
\quad-\frac{r}{T_{\max }}\left(\widehat{T}^{T} \widetilde{T} P \Phi(t)+\widehat{T}^{T} \widetilde{I} P \Phi(t)\right) \\
\quad-K_{1} \widehat{V}^{T} \widetilde{T} P \Phi(t) .
\end{aligned}
$$

Similarly, for (24b), we have

$$
\begin{aligned}
\int_{0}^{t} \dot{I}(\theta) \mathrm{d} \theta & =I(t)-I(0) \\
& =\widehat{I}^{T} \Phi(t)-I_{0}^{T} \Phi(t)
\end{aligned}
$$

by using (28a) and (28b) for the right-hand side of (24b), one can obtain

$$
\begin{aligned}
\int_{0}^{t}[ & \left.K_{2} V(\theta-1) T(\theta-1)-\mu_{I} I(\theta)\right] \mathrm{d} \theta \\
= & \int_{0}^{1}\left[K_{2} V(\theta-1) T(\theta-1)\right] \mathrm{d} \theta \\
& \quad+\int_{1}^{t}\left[K_{2} V(\theta-1) T(\theta-1)\right] \mathrm{d} \theta-\int_{0}^{t} \mu_{I} I(\theta) \mathrm{d} \theta
\end{aligned}
$$

$$
\begin{aligned}
= & \int_{0}^{1}\left(K_{2} \times 2000 \times 120\right) \mathrm{d} \theta \\
& +\int_{1}^{t} K_{2} \widehat{V}^{T} \phi(\theta-1) \Phi^{T}(\theta-1) \widehat{T} \mathrm{~d} \theta \\
& -\int_{0}^{t} \mu_{I} \widehat{I}^{T} \Phi(\theta) \mathrm{d} \theta \\
= & M_{1}+M_{2}-M_{3},
\end{aligned}
$$

where $M_{1}=\int_{0}^{1}\left(K_{2} \times 2000 \times 120\right) \mathrm{d} \theta, M_{2}=\int_{1}^{t} K_{2} \widehat{V}^{T} \phi(\theta-$ 1) $\Phi^{T}(\theta-1) \widehat{T} \mathrm{~d} \theta$, and $M_{3}=\int_{0}^{t} \mu_{I} \widehat{I}^{T} \Phi(\theta) \mathrm{d} \theta$.

To compute $M_{1}$, we consider $b=K_{2} \times 2000 \times 120$, which is approximated by Fourier series as

$$
b=B^{T} \Phi(t)
$$

where $B$ is the Fourier series coefficient vector of $b$ in the interval $I$ and $B=[b, 0, \ldots, 0,0, \ldots, 0]^{T}$.

By using (13) and (34), we have

$$
M_{1}=\int_{0}^{1} B^{T} \Phi(\theta) \mathrm{d} \theta=B^{T} \int_{0}^{1} \Phi(\theta) \mathrm{d} \theta=B^{T} Z \Phi(t) .
$$

To find $M_{2}$, one can use (12), (21), and (15). Hence,

$$
\begin{aligned}
M_{2} & =\int_{1}^{t} K_{2} \widehat{V}^{T} \phi(\theta-1) \Phi^{T}(\theta-1) \widehat{T} \mathrm{~d} \theta \\
& =K_{2} \widehat{V}^{T} \widetilde{T} \int_{1}^{t} \Phi(\theta-1) \mathrm{d} \theta \\
& \cong K_{2} \widehat{V}^{T} \widetilde{T} P \Phi(t-1) \cong K_{2} \widehat{V}^{T} \widetilde{T} P S_{1} \Phi(t)
\end{aligned}
$$

Similarly,

$$
M_{3}=\int_{0}^{t} \mu_{I} \widehat{I}^{T} \Phi(\theta) \mathrm{d} \theta=\mu_{I} \widehat{I}^{T} P \Phi(t) .
$$

Finally, by considering $M_{1}, M_{2}$, and $M_{3}$, we have

$$
\begin{aligned}
\int_{0}^{t} & {\left[K_{2} V(\theta-1) T(\theta-1)-\mu_{I} I(\theta)\right] \mathrm{d} \theta } \\
& =B^{T} Z \Phi(t)+K_{2} \widehat{V}^{T} \widetilde{T} P S_{1} \Phi(t)-\mu_{I} \widehat{I}^{T} P \Phi(t) .
\end{aligned}
$$

Now, we equalize (32) and (38); from the two sides of (24b),

$$
\begin{aligned}
\widehat{I}^{T} \Phi(t)-I_{0}^{T} \Phi(t)= & B^{T} Z \Phi(t)+K_{2} \widehat{V}^{T} \widetilde{T} P S_{1} \Phi(t) \\
& -\mu_{I} \widehat{I}^{T} P \Phi(t) .
\end{aligned}
$$

Similarly, by integrating (24c) from 0 to $t$, we obtain

$$
\begin{aligned}
\widehat{V}^{T} \Phi(t)-V_{0}^{T} \Phi(t)= & N_{1} \mu_{b} \widehat{U}^{T} \widetilde{I} P \Phi(t)-K_{1} \widehat{V}^{T} \widetilde{T} P \Phi(t) \\
& -\mu_{V} \widehat{V}^{T} P \Phi(t) .
\end{aligned}
$$




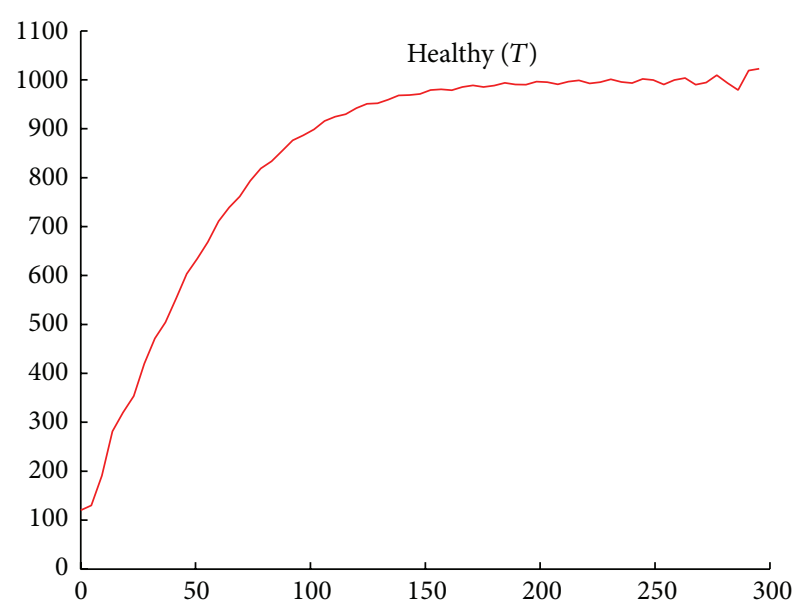

(a) $\mathrm{CD} 4^{+} \mathrm{T}$-cells $(T)$

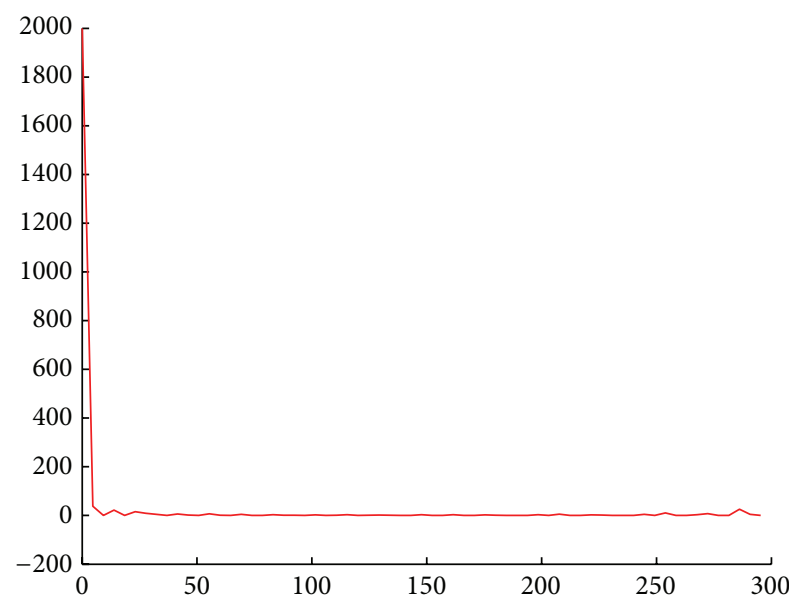

(c) Viral load $(V)$

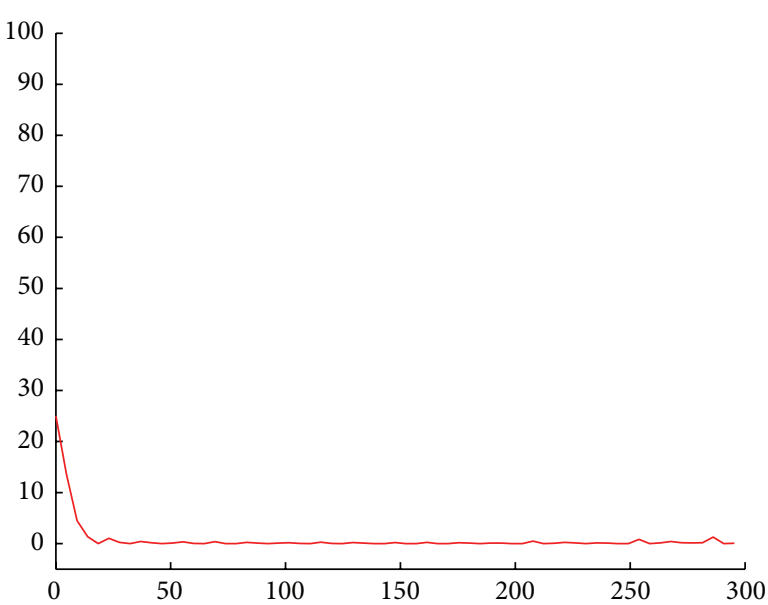

(b) Infected $\mathrm{CD} 4^{+} \mathrm{T}$-cells (I)

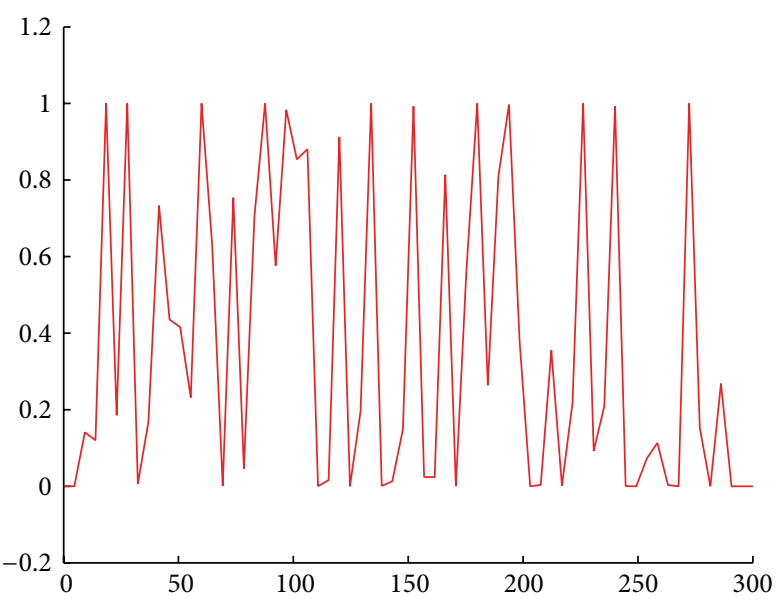

(d) Control (U)

Figure 1: The suboptimal $T(t), I(t), V(t)$, and $u(t)$.

Eliminating $\Phi(t)$ from (31), (39), and (40) results in the following equations:

$$
\begin{gathered}
\widehat{T}^{T}-T_{0}^{T}-S^{T} P-\left(r-\mu_{T}\right) \widehat{T}^{T} P \\
+\frac{r}{T_{\max }}\left(\widehat{T}^{T} \widetilde{T} P+\widehat{T}^{T} \widetilde{I} P\right)+K_{1} \widehat{V}^{T} \widetilde{T} P=0, \\
\widehat{I}^{T}-I_{0}^{T}-B^{T} Z-K_{2} \widehat{V}^{T} \widetilde{T} P S_{1}+\mu_{I} \widehat{I}^{T} P=0, \\
\widehat{V}^{T}-V_{0}^{T}-N_{1} \mu_{b} \widehat{U}^{T} \widetilde{I} P+K_{1} \widehat{V}^{T} \widetilde{T} P+\mu_{V} \widehat{V}^{T} P=0 .
\end{gathered}
$$

To apply the constraint $0 \leq u(t) \leq 1$, in (24h), one can first discretize the time interval into $(M+1)$ grid points as follows:

$$
t_{j}=t_{0}+h j, \quad j=0,1,2, \ldots, M,
$$

where $h=\left(\left(t_{f}-t_{0}\right) / M\right)$. Thus, $0 \leq u(t) \leq 1$ can be rewritten as

$$
0 \leq \widehat{U}^{T} \Phi\left(t_{j}\right) \leq 1, \quad j=0,1,2, \ldots, M
$$

Similarly, for $T(t), I(t)$, and $V(t) \geq 0$, we have

$$
\begin{array}{r}
\widehat{T}^{T} \Phi\left(t_{j}\right) \geq 0, \quad \widehat{I}^{T} \Phi\left(t_{j}\right) \geq 0, \quad \widehat{V}^{T} \Phi\left(t_{j}\right) \geq 0, \\
j=0,1,2, \ldots, M .
\end{array}
$$

Finally, the optimal control problem now is reduced to

$$
\begin{array}{ll}
\operatorname{Max} & J(\widehat{T}, \widehat{U})=\widehat{T}^{T} W-50\left(A^{T} W+\widehat{U}^{T} D \widehat{U}-2 \widehat{U}^{T} W\right) \\
\text { s.t. } & \widehat{T}^{T}-T_{0}^{T}-S^{T} P-\left(r-\mu_{T}\right) \widehat{T}^{T} P \\
& +\frac{r}{T_{\max }}\left(\widehat{T}^{T} \widetilde{T} P+\widehat{T}^{T} \widetilde{I} P\right)+K_{1} \widehat{V}^{T} \widetilde{T} P=0, \\
& \widehat{I}^{T}-I_{0}^{T}-B^{T} Z-K_{2} \widehat{V}^{T} \widetilde{T} P S_{1}+\mu_{I} \widehat{I}^{T} P=0, \\
& \widehat{V}^{T}-V_{0}^{T}-N_{1} \mu_{b} \widehat{U}^{T} \widetilde{I} P+K_{1} \widehat{V}^{T} \widetilde{T} P+\mu_{V} \widehat{V}^{T} P=0, \\
& 0 \leq \widehat{U}^{T} \Phi\left(t_{j}\right) \leq 1, \quad j=0,1,2, \ldots, M .
\end{array}
$$

Now, one can use various toolboxes like Matlab software to solve the above problem. The parameters of viral spread in 
TABLE 1: Parameters for viral spread in model (24a)-(24h).

\begin{tabular}{llc}
\hline Parameters & Values \\
\hline$\mu_{T}$ & Natural death rate of CD4 T-cells & 0.02 \\
$\mu_{I}$ & Blanket death rate of infected CD4 T-cells & 0.26 \\
$\mu_{b}$ & Lytic death rate for infected cells & 0.24 \\
$\mu_{V}$ & Death rate of free virus & 2.4 \\
$K_{1}$ & Rate by which CD4 T-cells become & $2.4 \times 10^{-5}$ \\
& infected with virus \\
$K_{2}$ & Rate by which infected cells become & $2 \times 10^{-5}$ \\
$r$ & active & 0.03 \\
$N_{1}$ & Growth rate of CD4 T-cell population \\
$T_{\max }$ & Number of virions produced by infected & 1000 \\
$s$ & CD4 T-cells & 1500 \\
\hline
\end{tabular}

model (24a), (24b), (24c), (24d), (24e), (24f), (24g), and (24h) are presented in Table 1.

The results of this optimization problem for $r=45$ and $M=65$ are depicted in Figure 1. As can be seen, utilizing the treatment increases the CD4 ${ }^{+}$T-cells (T) (Figure 1(a)) and decreases the infected CD4 ${ }^{+}$T-cells $(I)$ (Figure $1(\mathrm{~b})$ ) and viral load $(V)$ (Figure 1(c)).

\section{Conclusion}

In this paper, we consider a delayed mathematical model describing HIV infection of $\mathrm{CD} 4{ }^{+}$T-cells during therapy.

Our objective is to minimize the cost of treatment and to maximize the uninfected $\mathrm{CD} 4^{+} \mathrm{T}$-cells. We use Fourier series approximation in order to solve the so-called mathematical model. By applying operational matrices for integration, product, and delay, the nonlinear optimal control model is reduced to a set of nonlinear algebraic equations. Since the set of sines and cosines is orthogonal, the operational matrices contain many zero elements. Hence, it makes the method computationally easy and attractive. The results show the efficiency of the method.

\section{Conflict of Interests}

The authors declare that there is no conflict of interests regarding the publication of this paper.

\section{Acknowledgment}

This research was in part supported by a grant from the Center of Excellence on Modelling and Control Systems (CEMCS).

\section{References}

[1] M. Jamshidi and C. M. Wang, "A computational algorithm for large-scale nonlinear time-delay systems," IEEE Transactions on Systems, Man, and Cybernetics, vol. 14, no. 1, pp. 2-9, 1984.
[2] K. Hattaf and N. Yousfi, "Dynamics of HIV infection model with therapy and cure rate," International Journal of Tomography and Statistics, vol. 16, no. 11, pp. 74-80, 2011.

[3] K. Hattaf, N. Yousfi, and A. Tridane, "Mathematical analysis of a virus dynamics model with general incidence rate and cure rate," Nonlinear Analysis: Real World Applications, vol. 13, no. 4, pp. 1866-1872, 2012.

[4] A. S. Perelson and P. W. Nelson, "Mathematical analysis of HIV1 dynamics in vivo," SIAM Review, vol. 41, no. 1, pp. 3-44, 1999.

[5] A. S. Perelson, A. U. Neumann, M. Markowitz, J. M. Leonard, and D. D. Ho, "HIV-1 dynamics in vivo: virion clearance rate, infected cell life-span, and viral generation time," Science, vol. 271, no. 5255, pp. 1582-1586, 1996.

[6] X. Zhou, X. Song, and X. Shi, "A differential equation model of HIV infection of CD4+ T-cells with cure rate," Journal of Mathematical Analysis and Applications, vol. 342, no. 2, pp. 1342-1355, 2008.

[7] H. Zhu and X. Zou, "Dynamics of a HIV-1 infection model with cell-mediated immune response and intracellular delay," Discrete and Continuous Dynamical Systems B, vol. 12, no. 2, pp. 511-524, 2009.

[8] K. Hattaf and N. Yousfi, "Two optimal treatments of HIV infection model," World Journal of Modelling and Simulation, vol. 8, no. 1, pp. 27-36, 2012.

[9] K. R. Fister, S. Lenhart, and J. S. McNally, "Optimizing chemotherapy in an HIV model," Electronic Journal of Differential Equations, vol. 1998, 12 pages, 1998.

[10] H. R. Joshi, "Optimal control of an HIV immunology model," Optimal Control Applications \& Methods, vol. 23, no. 4, pp. 199213, 2002.

[11] J. Karrakchou, M. Rachik, and S. Gourari, "Optimal control and infectiology: application to an HIV/AIDS model," Applied Mathematics and Computation, vol. 177, no. 2, pp. 807-818, 2006.

[12] D. Kirschner, S. Lenhart, and S. Serbin, "Optimal control of the chemotherapy of HIV," Journal of Mathematical Biology, vol. 35, no. 7, pp. 775-792, 1997.

[13] H.-D. Kwon, "Optimal treatment strategies derived from a HIV model with drug-resistant mutants," Applied Mathematics and Computation, vol. 188, no. 2, pp. 1193-1204, 2007.

[14] R. Culshaw, S. Ruan, and R. J. Spiteri, "Optimal HIV treatment by maximising immune response," Journal of Mathematical Biology, vol. 48, no. 5, pp. 545-562, 2004.

[15] R. V. Culshaw and S. Ruan, "A delay-differential equation model of HIV infection of $\mathrm{CD} 4^{+}$T-cells," Mathematical Biosciences, vol. 165, no. 1, pp. 27-39, 2000.

[16] W. Chen and Y. Shih, "Shift Walsh matrix and delay-differential equations," IEEE Transactions on Automatic Control, vol. 23, no. 6, pp. 1023-1028, 1978.

[17] G. P. Rao and T. Srinivasan, "Analysis and synthesis of dynamic systems containing time delays via block-pulse functions," Proceedings of the Institution of Electrical Engineers, vol. 125, no. 10, pp. 1064-1068, 1978.

[18] F. Kung and H. Lee, "Solution and parameter estimation in linear tim e-invariant delay systems using Laguerre polynomial expansion," Journal of Dynamic Systems, Measurement and Control, vol. 105, no. 4, pp. 297-301, 1983.

[19] L. Lee and F. Kung, "Shifted Legendre series solution and paramet er estimation of linear delayed systems," International Journal of Systems Science, vol. 16, no. 10, pp. 1249-1256, 1985.

[20] I. R. Horng and J. H. Chou, "Analysis, parameter estimation and optimal control of time-delay systems via Chebyshev series," 
International Journal of Control, vol. 41, no. 5, pp. 1221-1234, 1985.

[21] M. Razzaghi and M. Razzaghi, "Fourier series direct method for variational problems," International Journal of Control, vol. 48, no. 3, pp. 887-895, 1988.

[22] Gh. Ghanbari and M. H. Farahi, "Optimal control strategy for a HIV infection model via Fourier series," Journal of Applied Mathematics, Statistics and Informatics, vol. 9, no. 2, pp. 87-94, 2014.

[23] D. H. Eller, J. K. Aggarwal, and H. T. Banks, "Optimal control of linear time-delay systems," IEEE Transactions on Automatic Control, vol. 14, pp. 678-687, 1969.

[24] N. N. Krasovskii, Stability of Motion: applications of Lyapunov's second Method to Differential Systems and Equations with Delay, 1963.

[25] R. Loxton, K. L. Teo, and V. Rehbock, "An optimization approach to state-delay identification," IEEE Transactions on Automatic Control, vol. 55, no. 9, pp. 2113-2119, 2010.

[26] L. Göllmann, D. Kern, and H. Maurer, "Optimal control problems with delays in state and control variables subject to mixed control-state constraints," Optimal Control Applications and Methods, vol. 30, no. 4, pp. 341-365, 2009. 

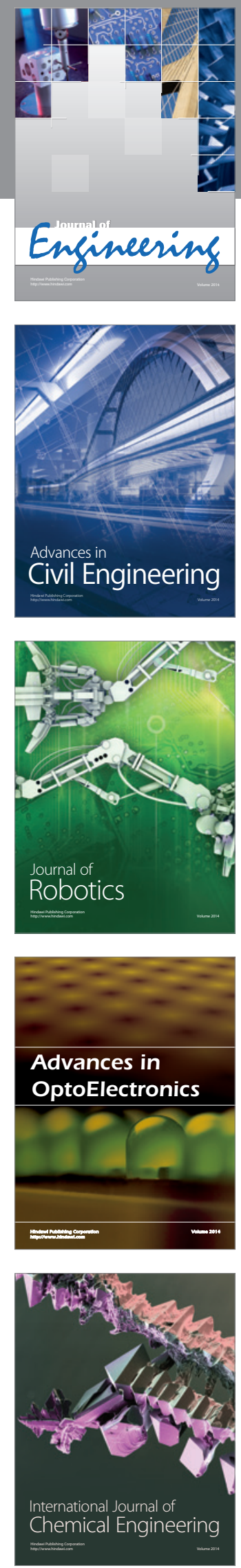

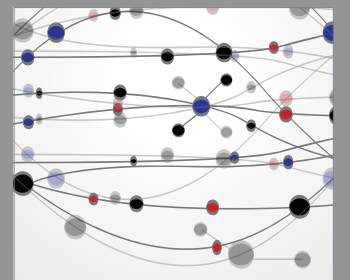

The Scientific World Journal
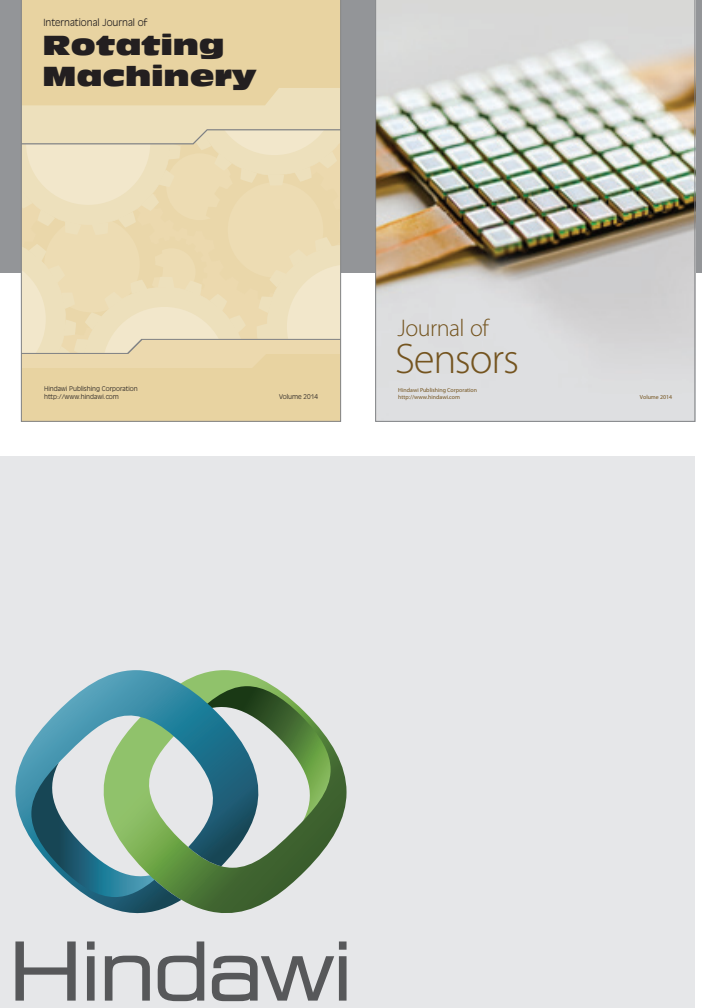

Submit your manuscripts at http://www.hindawi.com
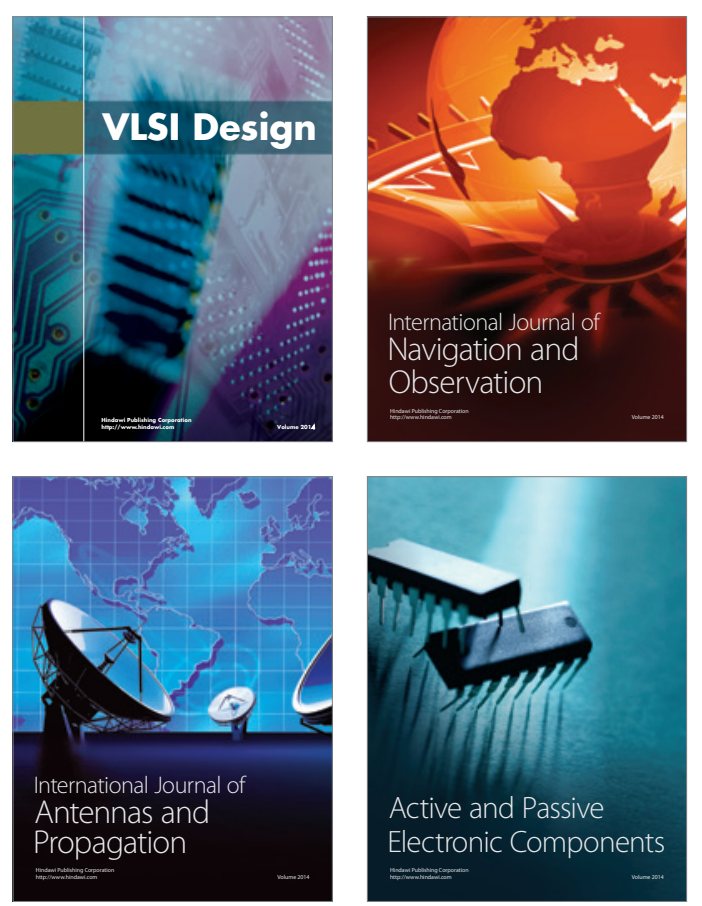
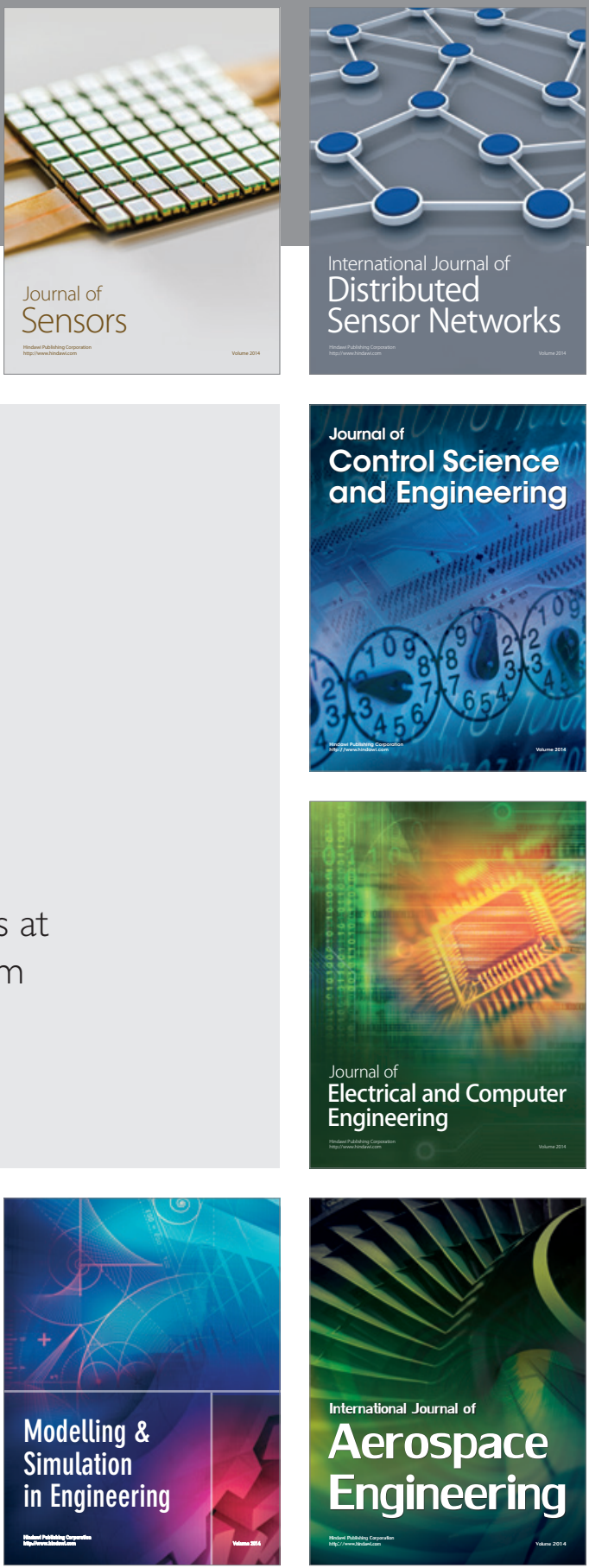

Journal of

Control Science

and Engineering
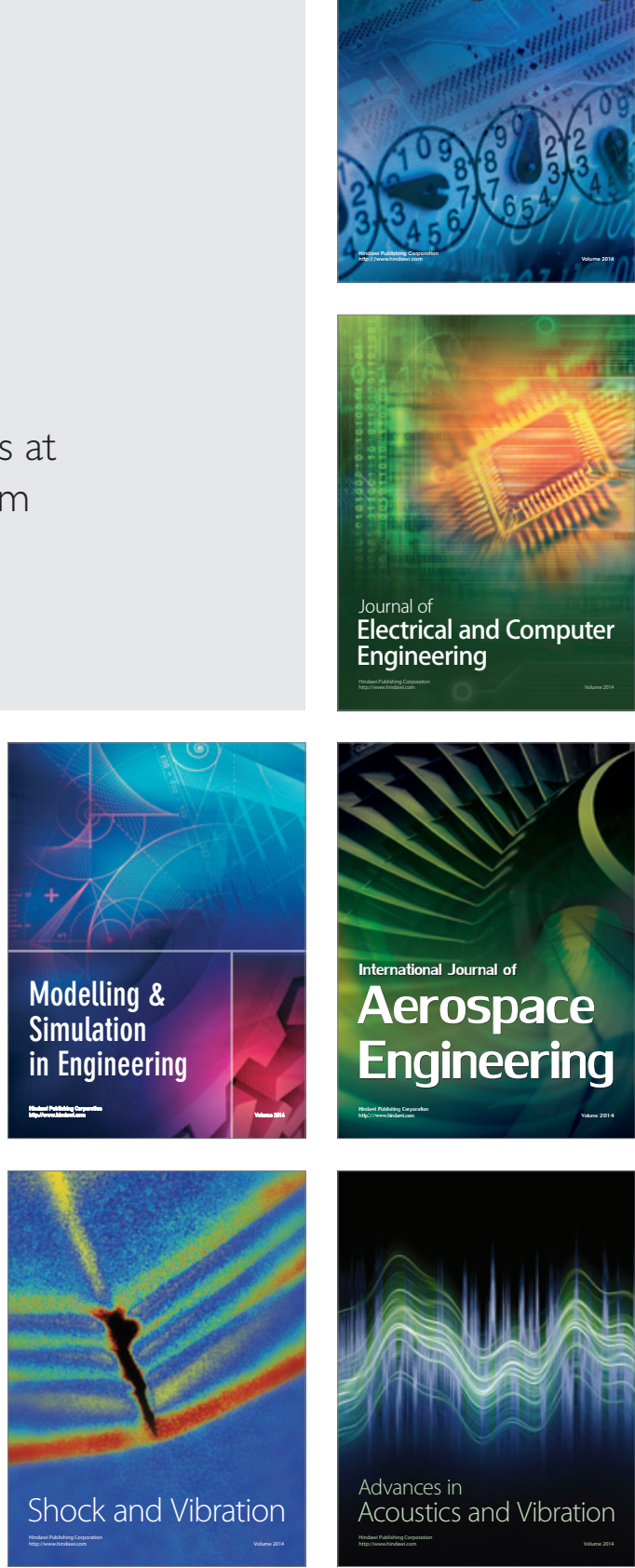